\title{
Transoral laser surgery using Optiflow high-flow nasal cannula oxygenation
}

\author{
S Flach, A Elhoweris, S Majumdar, J Manickavasagam
}

Department of Otolaryngology and Head \& Neck Surgery, Ninewells Hospital, Dundee, U.K.

\section{Introduction}

Endoscopic surgical treatment of oropharyngeal conditions relies on good access and adequate oxygenation of the patient which can be achieved by a variety of means. Methods to provide oxygenation during tubeless field anaesthesia include: supraglottic, transglottic and transtracheal jet ventilation and low-flow oxygen delivery as part of a spontaneously ventilating technique. Oxygen delivery via high-flow nasal cannulae (HFNC) has been increasingly adopted in adult patients. Controversy exists about the fire risks of laser surgery in the presence of supplementary oxygen.

We describe our experience with $\mathrm{CO}_{2}$ laser excision for a variety of cases using the HFNC oxygen delivery system Optiflow.

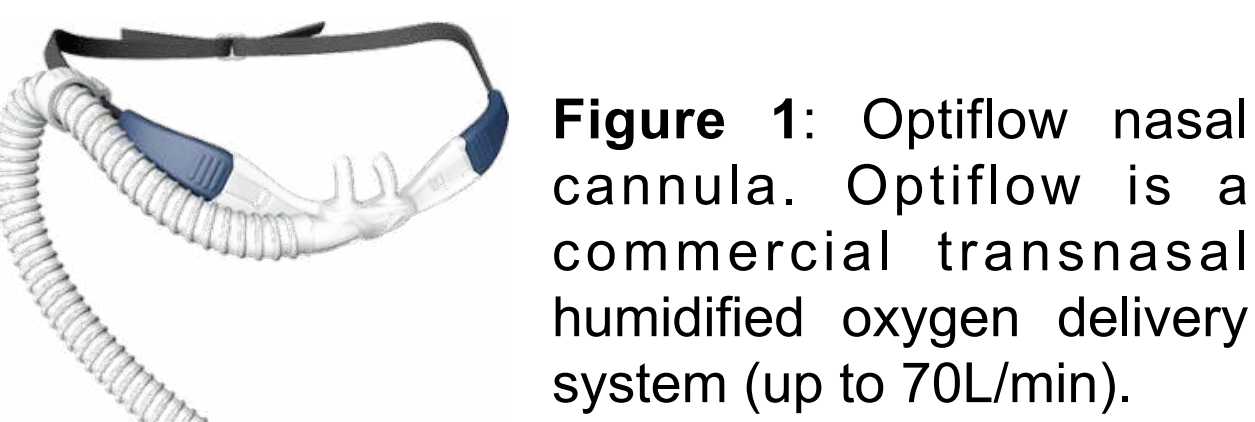

Source: https://tri-medmedical.com/products/fisher-payke-

\section{Methods}

We retrospectively reviewed the medical records of patients who underwent $\mathrm{CO}_{2}$ laser-aided excision under Optiflow HFNC airway management at our institution during 01/2015-02/2018.

\section{Conclusion}

Optiflow HFNC provides a safe and efficient oxygenation system during transoral endoscopic surgery of oropharyngeal conditions using $\mathrm{CO}_{2}$ lasers.

\section{Results: Summary of demographics}

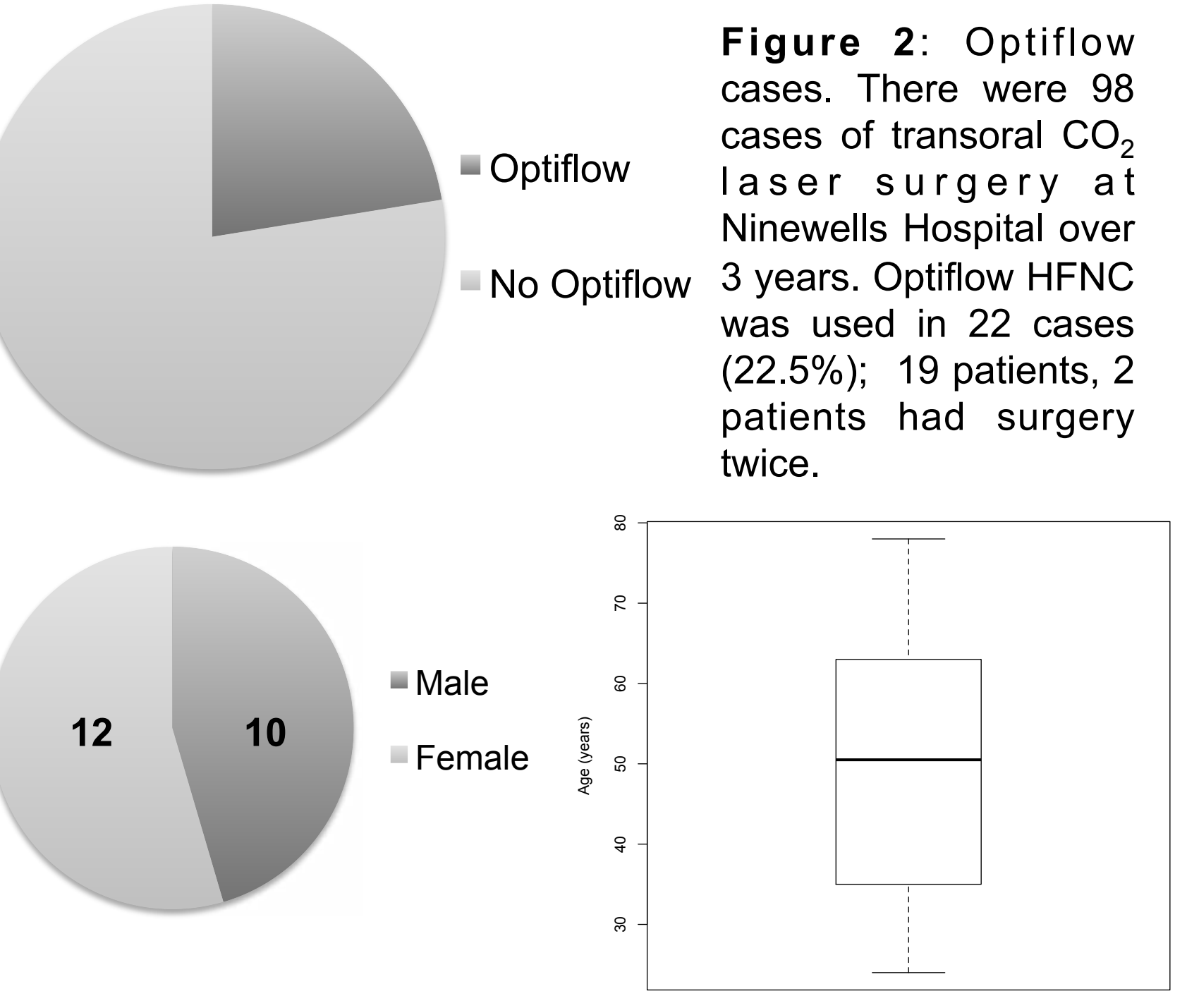

(a)

(b)

Figure 3: Demographics of Optiflow cases. (a) $45 \%$ male, $55 \%$ female. (b) The median age was 50.5 years (range $24-78$ years).

Results: Summary of Optiflow cases

Figure 4: Median Optiflow time. The median Optiflow time during surgery was 45min (range 25-180 $\mathrm{min}$ )

subglottic stenosis

cordectomy/cordotomy

laryngeal papilloma

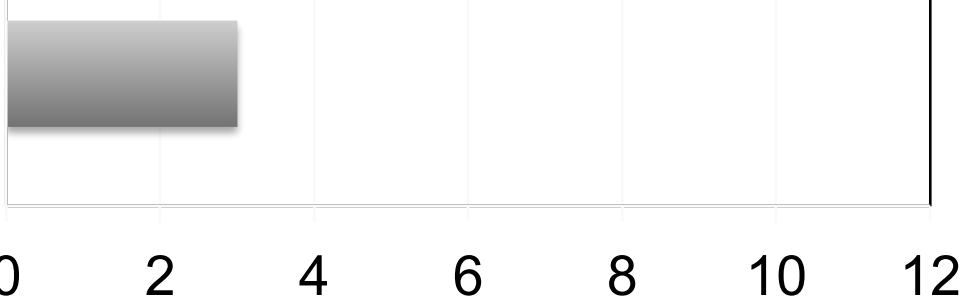

Figure 5: Summary of surgical cases where Optiflow was used.

\section{Results: Complications}

- 1 case of dilatation for subglottic stenosis had transient low saturations during balloon dilatation (rapid recovery).

- No other intra- or immediate post-operative complications recorded. No burns or fires. 